\title{
Acerca de los mecanismos de desconstrucción del orden social instituyente durante el momento adolescente $^{* 1}$
}

Alejandro Bilbao*2

Daniel Jofré*3

Los estudios desarrollados sobre la cuestión del súperyo en el momento adolescente, reconocen la posibilidad y la necesidad de distanciamiento del sujeto frente a esta figura, dejando no obstante abiertas las preguntas por los mecanismos de esta operación. En este artículo, se propone una lectura de esta problemática, mediante el análisis del vínculo entre el trabajo de posicionamiento adolescente frente al orden social instituyente y el trabajo de la sublimación en el sujeto. Con este propósito se propondrá comprender las posibilidades del sujeto de posicionarse éticamente frente a lo real, a partir de la construcción de un espacio subjetivo de transición, traducción y narración.

Palabras clave: Ética, sublimación, orden social instituyente, singularidad

\footnotetext{
${ }^{* 1}$ Este texto fue redactado dentro del marco del proyecto de investigación Ecosconicyt "Las formas de la individuación en el mundo contemporáneo". Investigación financiada por el consejo nacional de investigación científica y tecnológica del gobierno de Chile.

*2 Universidad Andres Bello (Santiago de Chile, Chile).

*3 Universidad Austral de Chile (Puerto Montt, Chile).
} 


\section{ARTIGOS}

\section{Introducción}

La cuestión del súperyo durante la adolescencia sitúa el trabajo de historización adolescente dentro del espacio que se abre entre los componentes ideales del Otro parental y el Otro social (Rassial, 1996; Gutton, 2002; Lesourd, 2004; Sauret, 2009). De este modo el trabajo de la adolescencia, se orienta hacia la validación de la división estructural del sujeto, la cual se desarrolla dentro de una dinámica en donde opera la renovación del movimiento de separación y alienación del sujeto al Otro (Haie y Douville, 2007; Haie y Rassial, 2008). La ambigüedad de este proceso reside en la dependencia del adolescente respecto de los ideales sociales, a través de los cuales, el adolescente encuentra la posibilidad de reconstruir su propia subjetividad, pero encuentra asimismo una nueva tendencia a la alienación.

En esta perspectiva, el presente artículo buscará comprender el vínculo entre el reconocimiento del carácter arbitrario de la ley social y la posibilidad de deconstruir los imperativos e ideales movilizados por el súperyo durante la adolescencia. Siguiendo este objetivo, la primera parte del artículo se dedica a abordar las ideas centrales propuestas por Jacques Lacan respecto de la ética y la sublimación, como también se presenta la perspectiva de J. Gagnepain (1991) y sus discípulos, respecto del carácter inestable y provisorio de los procesos de apropiación subjetiva durante la adolescencia. Durante la segunda parte del articulo, se desarrolla un análisis respecto de los mecanismos de deconstrucción del sujeto frente al Otro social. La argumentación se centra en la relación entre el empuje pulsional, la apropiación de la instancia de la Ley simbólica y la cuestión de la legitimidad de la ley social. A este respecto, se propone comprender las posibilidades éticas del sujeto adolescente como la creación de un espacio subjetivo de transición, el cual opera mediante la continua construcción de nuevas narrativas, individuales e intersubjetivas, que permiten la inscripción del sujeto dentro del cuerpo social.

Rev. Latinoam. Psicopat. Fund., São Paulo, 20 (3), 436-450, set. 2017 


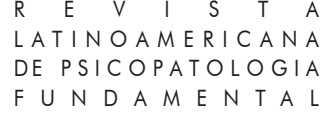

Primera Parte

\section{Lacan y el acto ético del sujeto}

Uno de los puntos más difíciles dentro de los esfuerzos por examinar la relación entre lo individual y lo colectivo dentro de la teoría psicoanalítica, esta representado por la posibilidad de considerar para el sujeto del inconsciente un espacio ético que sobrepase los márgenes del Otro. De hecho, como lo precisa Freud (1950[1895]/1978), es en el momento mítico de la primera satisfacción, dentro de la necesidad de la presencia de ayuda externa, donde se reconoce el nacimiento del deseo y la fuente primordial de la moral para el sujeto. Dentro de la dinámica de estructuración psíquica, el psicoanálisis freudiano reconoce en el encuentro con la Ley del Padre, la posibilidad para el niño de posicionarse frente al súperyo (1923-1925/1978a). En el transcurso de su obra, Freud no cesará en la reflexión sobre la relación del hombre y los ideales culturales (1921/1978b), introduciendo la tesis, que es en el vínculo entre el mundo pulsional y las exigencias culturales donde el hombre encuentra su más radical contradicción (1920/1978c).

La teoría del sujeto desarrollada por Lacan permite retomar los ejes fundamentales de los desarrollos freudianos respecto de la relación del hombre y el medio que lo constituye. Los trabajos de Lacan (1966; 1961-1962/2010) a este respecto introducen la contradicción fundamental del deseo humano en la imposibilidad de acceder a su satisfacción, haciendo emerger la diferencia, la repetición y la negatividad como el soporte último del vínculo entre el sujeto y el Otro. Asimismo, Lacan (1959-1960/1986) aborda la cuestión de la ética en psicoanálisis, invitando a reconocer que el espacio ético del sujeto esta constituido por el margen presente entre dos muertes. La muerte que reenvía nuevamente al hombre a su carácter biológico, y la muerte que representa la necesidad para el sujeto de fundar sus actos éticos sobrepasando los imperativos sociales de la sociedad dentro de la cual habita. Asumiendo esta comprensión del acto ético del sujeto, Lacan (1964/1973) discernirá el movimiento acéfalo de la pulsion, como un impulso tendiente a simbolizar lo real dentro de la experiencia del sujeto de lo inconsciente. Se fortalece de este modo una idea de lo humano, que reintroduce al sujeto dentro del conjunto de sus determinaciones, donde las significaciones e imaginarios socio históricos, representan una dimensión insoslayable del sujeto, pero no su totalidad. De esta forma, dentro de los desarrollos de Lacan, el acto ético del sujeto sobrepasa 


\section{ARTIGOS}

la dicotomía que diferencia lo individual de lo colectivo, de suerte que se subrayan las posibilidades de posicionamiento del sujeto frente a la falta del Otro y el rencuentro del sujeto con el vacío de lo real, orientando de este modo la reflexión a la relación entre lo real y la sublimación.

Ahora bien, desde la perspectiva psicoanalítica, la posibilidad de la sublimación participa de los movimientos del sujeto dentro de una dinámica donde confluyen sus determinaciones simbólicas y pulsionales, siendo contradictoria por ello, con un estado de plenitud posible de alcanzar en un momento de la vida (Mijolla-Mellor, 2009 y 2012). En esta perspectiva, como invita a pensar Viviana Saint-Cyr (2012), dentro de los procesos sublimatorios, se busca superar el registro imaginario de los objetos especulares, dirigiendo al sujeto hacia una otra Cosa, o más bien, hacia el vacío detrás de la Cosa. Se establece por consecuencia, la paradoja esencial de los procesos sublimatorios en la necesidad de superar lo imaginario a fin de rencontrar lo real, pero admitiendo enseguida la imposibilidad de la representación de lo real, en cuanto la Cosa (lo real) no puede sino ser ausente porque representa el vacío inherente a la articulación de los significantes. El proceso sublimatorio se aproxima, en este sentido, a un trabajo incesante de simbolización de lo real, dentro del cual se supone la posibilidad de situar otra cosa en el lugar de la Cosa.

\section{Jean Gagnepain: La apropiación subjetiva y el carácter contingente de la ley}

J. Gagnepain (1991) desarrolla un modelo teórico que tiene por objeto analizar la capacidad humana de habitar en el lenguaje, de construir instrumentos, de posicionarse históricamente y a nivel del deseo. Este modelo se constituye desde la diferenciación de tres tiempos lógicos : la condición natural, el análisis formal (instancia) y el momento de investidura positiva (performance). De esta forma, la dialéctica entre la instancia y la performance, por una parte, rompe con la condición natural del hombre, permitiendo las diferentes modalidades en donde se expresa la racionalidad humana, y por otra parte, reintroduce la negatividad como una fracción irresoluble por estas mediaciones del comportamiento cultural del hombre. En particular, el modelo de análisis propuesto en la dialéctica de la Persona, trabaja la contraposición dialéctica entre lo étnico y lo político, lo singular y lo universal. De suerte que en este registro se diferencia, $i$ ) la condición natural de la sexualidad y la procreación humana, ii) la institución del viviente humano dentro del orden social que lo orienta en el registro de la genitalidad y la paternidad, 


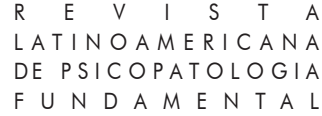

y iii) el trabajo de singularización del orden social mediante el reconocimiento de su historicidad. Por consiguiente, mediante este proceso dialéctico, el adolescente reorganiza su posicionamiento dentro de la sociedad, a través de la singularización de las disposiciones socio discursivas de la colectividad, permitiendo de este modo la apropiación del código social, a nivel de la lengua, del discurso y de las acciones de individuo (Gagnepain, 1991; Brackelaire, 1995; Quentel, 2011).

A esta luz, dentro del trabajo que permite en el momento adolescente la apropiación del orden social, se subraya la profunda inestabilidad de este proceso de singularización, de suerte este proceso también representará, el momento en el curso del cual el adolescente, se debe confrontar a la impropiedad de sus esfuerzos por donar un sentido a su propia vida. En este sentido, el encuentro del adolescente con la contingencia del orden social, lo reconduce también al carácter siempre provisorio del decir humano.

La propuesta aquí desarrollada aspira a retener estas precisiones y observar la importancia que tiene vincular este proceso de singularización de la Persona al trabajo de simbolización de lo real del sujeto durante la adolescencia. En este sentido, se propone concebir la tension permanente y el carácter inestable del proceso de apropiación subjetiva, como una forma de pensar las simbolizaciones de los adolescentes frente al carácter impenetrable de lo real. Por cierto, respecto de las nociones de sujeto y Persona, es conveniente no obstante indicar, que éstas guardan su propio horizonte conceptual de inteligibilidad. La noción de sujeto, encuentra su punto de validación en la aprehensión de las simbolizaciones humanas por la lógica de lo inconsciente, la cual inscribe el decir de toda cría humana en el horizonte del deseo y la falta. La noción de persona, como se ha indicado, encuentra su punto de aplicación, en el análisis de la relación entre el individuo y los fenómenos sociales, a partir del examen de la dialéctica entre lo singular y lo universal.

Se propone pues, reconocer dentro del trabajo de la adolescencia la apertura a un espacio de simbolización, dentro del cual es posible crear nuevas posiciones subjetivas, a partir del reconocimiento de la participación del sujeto dentro del devenir histórico de las figuras ideales constitutivas del Otro. Así, a diferencia de los procesos sublimatorios de la infancia, dentro de los cuales la promesa de completitud ideal permite encaminar un proyecto para el sujeto; en el momento adolescente, se puede inferir, el único proyecto posible para el sujeto consistiría en comprender la contingencia de los elementos fundadores de la propia subjetividad. Siendo factible en consecuencia sostener, que la relación del sujeto con la Ley durante la adolescencia 


\section{ARTIGOS}

redobla la paradoja entre los esfuerzos por tocar la Cosa y la necesidad de su representación, orientándose por lo tanto, hacia el horizonte dinámico e inestable, de los procesos sublimatorios.

\section{Segunda PARTe}

\section{El momento adolescente: deconstrucción, transmisión y singularización}

El tiempo de la adolescencia permite discernir el punto de quiebre respecto de la lógica del Otro parental, de hecho, la apertura a la historia y la necesidad de singularización conlleva la posibilidad de diferenciar entre la Ley, en tanto que principio, y los contenidos e imperativos que moviliza. Este aspecto del proceso adolescente esta relacionado con una cuestión fundamental de la relación del hombre con el Ideal: la posibilidad humana de deconstruir las disposiciones e imperativos de la Ley.

Sobre esta cuestión, los estudios psicoanalíticos sobre el súperyo adolescente (Rassial, 1999; Dufour, 2003; Forget, 2005; Lesourd, 2006; Douville, 2014), sitúan las posibilidades del sujeto de deconstruir los imperativos del Otro en el reconocimiento de la falta simbólica y lo real que organiza la relación del sujeto al Otro. Se trata pues, ante todo, de comprender que el poderío de los mecanismos que ligan al adolescente a los ideales e imperativos sociales, reside en el empuje pulsional que fuerza por introducir al sujeto en una dinámica, en donde los objetos imaginarios propuestos al deseo, refuerzan la dependencia del sujeto al Otro, cristalizando las posiciones y las posibilidades creativas del sujeto frente a lo real (Sauret, 2008). Respecto de la potencialidad humana de deconstruir los imperativos de la Ley, la antropología clínica de Jean Gagnepain apuesta por diferenciar el plano de la relación del hombre con la Ley, de la capacidad del hombre de analizar su propio comportamiento, estableciendo para ello diferentes posibilidades de restricción y habilitación del deseo (Gagnepain, 1991; Gauchet, 2009). Se propone pues, la diferenciación entre el plan de lo ético-moral y la dialéctica entre el individuo y lo social, distinguiendo el dominio de la institución en tanto que determinismo social sobre el individuo y el dominio de la reglamentación del deseo. En lo fundamental, como apunta Gagnepain (2010), esta diferenciación busca distinguir la doble capacidad humana de analizar el propio deseo y al mismo tiempo someter a análisis la ley que lo inscribe 


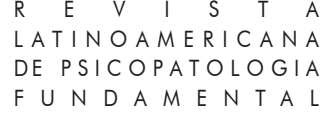

dentro de un código. En este sentido, un aspecto central de esta diferenciación radica en los continuos recortes que se establecen entre estos dos planos, de hecho, el plano de lo social puede operar sobre el ámbito de la reglamentación del deseo, legalizando lo legitimo, y al contrario, tanto a nivel individual como colectivo, el reconocimiento del carácter arbitrario de la Ley, conlleva la posibilidad de su puesta en juicio mediante el cuestionamiento de la legitimidad de la legalidad social. Se opone por consecuencia, la capacidad del orden socio simbólico de introducir mecanismos específicos de construcción de moralidades y normas especificas de derecho, las que conformarán la experiencia moral del individuo dentro de la comunidad, a la capacidad de deconsturir el dominio de la Ley, poniendo en cuestión los imperativos sociales.

Ahora bien, asumiendo una y otra de estas perspectivas, todo parece apuntar que la cuestión fundamental respecto de la relación entre el sujeto adolescente y los ideales sociales presentes en el súper yo adolescente, radicaría en la necesidad de donar una respuesta la siguiente cuestión: ¿Cómo un proceso social e intersubjetivo de cuestionamiento de la legitimidad de las leyes de la comunidad, se vincularía a los mecanismos que ligan al sujeto, a una economía pulsional y psíquica, de dependencia frente al Otro? Frente a esta cuestión,

442 sería posible convenir (i) que la apertura del sujeto al cuestionamiento de los significantes amo de su entorno simbólico, permite al adolescente posicionar su propia subjetividad frente al carácter contingente de su pertenencia social. (ii) Siendo este proceso de apropiación un proceso dialéctico de rencuentro con el Otro y la falta constituyente, el posicionamiento del sujeto se conformaría de acuerdo a un continuo esfuerzo de simbolización; que le permitirá desarrollar un trabajo deconstructivo de los imperativos súperyoicos a través de las cuales mide sus actos como sujeto. Por consecuencia, (iii) este proceso crítico, creativo y de reencuentro con el otro, que permite la desconstrucción de los imperativos e ideales del súperyo, se funda en la inestabilidad y la singularidad del trabajo de la negatividad inherente a los procesos sublimatorios.

\section{Los nombres del padre, lo ético y lo social}

Se puede formular la idea de que dentro del momento adolescente, se conforma la posibilidad de restablecer el vínculo entre lo social y lo ético. Se trataría por cierto, de pensar este momento de simbolización adolescente como una dinámica, dentro de la cual la apropiación del orden social se desarrolla a partir de un proceso de historizacion, el mismo sostenido desde 


\section{ARTIGOS}

lo real. En este sentido, a partir del momento adolescente el sujeto, por una parte, se abre la posibilidad de renovar los códigos sociales en acuerdo con los cuales se organizan y son legitimas las acciones de los individuos dentro de la comunidad, y por otra parte, el sujeto puede reconocer, en el empuje incesante del mundo pulsional, el fundamento ético de sus acciones.

Ahora bien, en este punto cabe señalar, que la ética del psicoanálisis nos orienta hacia una lectura que se centra en la relación entre el placer y la ética, pero específicamente en la imposibilidad constitutiva de esta relación. Lo cual, entre otras cosas, hace de la ética del psicoanálisis, un ética antagonista respecto de aquellas que orientan la interrogante respecto del bienestar humano en el horizonte de un soberano Bien. Al contrario, como lo indica Freud en su estudio sobre "El malestar en la cultura" (1920/1978c), la problemática central de la relación del hombre y su búsqueda de bienestar, se concentra en la imposible realización de esta tarea, en cuanto, independientemente de las técnicas del saber-vivir que puedan ser utilizadas, el displacer retorna a la experiencia humana, bajo la modalidad súperyoica de la exigencia de bienestar. Siendo por ello posible indicar, que desde el punto de vista de las dinámicas subjetivas, la ética del psicoanálisis demanda al sujeto un ejercicio continuo de deconstrucción de las exigencias del súperyo, siendo contradictoria por esta razón, respecto de los ideales sociales, como por ejemplo, el ideal de autenticidad o no dependencia. Cabe subrayar, por otra parte, que el análisis que realiza Lacan sobre la ética del psicoanálisis (1959-1960/1986) se fundamenta en la separación originaría del viviente humano con la Cosa, el das Ding freudiano, lo cual le permite remarcar el carácter de lo imposible y la falta, a partir de la disyunción entre la condición de sujeto y el carácter biológico del hombre, su condición natural. Se debe advertir asimismo que esta separación del hombre y la Cosa, producto de la Ley del lenguaje, se re articula en el horizonte cultural judeo-cristiano, con el monoteísmo y a la relación del sujeto con el Padre y la culpa. En este sentido, Lacan (1961-1962/2010) retoma la cuestión del mito originario del Padre primordial desarrollado por Freud y lo incorpora a la experiencia humana en el momento de aprehensión del infante en el campo de la letra, producto de la marca del significante. Siendo esta identificación primordial y originaria al rasgo unario, la que orientará la dinámica del sujeto a nivel del deseo y instituirá su relación de dependencia al saber del Otro. En suma, la relación del hombre y sus aspiraciones éticas, reenvía en el análisis de Freud y Lacan, por una parte, a la función intrapsíquica del súperyo, al carácter imperativo de la Ley y a la dependencia del sujeto respecto de ella; pero por otra parte, también reenvía 


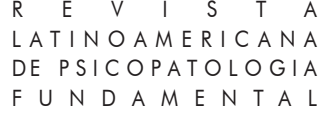

al ámbito socio-cultural, en donde la ley del Padre y los ideales sociales, se recubren e invisten del poderío de la Ley del lenguaje.

Avancemos pues siguiendo estas dos vertientes. Respecto de la cuestión del vínculo entre la Ley del lenguaje, la ley del Padre y los ideales sociales, los estudios psicoanalíticos sobre la adolescencia, desarrollan una hipótesis que procede del siguiente modo: i) durante la infancia la Ley del lenguaje, que excluye para el infante humano el goce de la Cosa, se reviste necesariamente bajo los ropajes de la figuras paternas de terceridad; sin embargo, ii) en la adolescencia, la Ley del lenguaje y la Ley del Otro social, se tornan disjuntas a causa del encuentro con lo polo femenino de la sexuación (Lesourd, 2002), lo cual permite la declinación el poderío de la ley del padre, a favor de los ideales sociales, siendo en este espacio entre dos leyes (Rassial, 1996) en donde se desarrolla el trabajo de la adolescencia y explota su conflictividad. Por otra parte, partiendo esta vez de la diferenciación entre el carácter intrapsíquico del súperyo y su aspecto sociocultural, Jean Gagnepain (2010), como hemos visto, remarca la importancia clínica de distinguir entre los mandatos del Otro social, encarnados en la figura del súperyo adolescente, y por lo tanto, presentes como vivencia 444 psíquica para el adolescente, y la cuestión de la ley como fundamento del lazo social. En este sentido, se subraya la importancia de proceder hacia una examen crítico, individual pero también intersubjetivo, respecto de la legitimidad de la ley social, y al mismo tiempo, se subraya la necesidad del adolescente de analizar su propio deseo y sus actuaciones, propiciando una distancia respecto de los imperativos súperyoicos, a momento de restringir su propio deseo y de permitir su satisfacción.

Se conforma de esta modo, una compresión de las posibilidades éticas del sujeto, que remarca el proceso de posicionamiento subjetivo frente a la ley social, a través del análisis de las exigencias súperyoicas, pero que al mismo tiempo insiste en el reconocimiento de la falta y lo imposible asociada a la Ley del lenguaje. Siguiendo estas lecturas es factible pensar: la negatividad de lo real durante el momento adolescente, orienta las simbolizaciones del sujeto hacia una constante deconstrucción de los imperativos del Otro social y a desarrollar continuos esfuerzos de creación y apropiación, a partir de los cuales conformar una narrativa, intrapsíquica pero también social e intersubjetiva, capaz de orientar el propio deseo y de posicionar la propia subjetividad en el horizonte del discurso y el lazo social.

Este supuesto permite reconocer, en la construcción de nuevas referencias de terceridad, un aspecto fundamental del proceso de simbolización 
adolescente, de suerte que subjetivar la adolescencia significaría también la posibilidad de ligar el sujeto al mundo social, esto de una manera apta a integrar y transmitir, las referencias subjetivas de la infancia dentro de las nuevas disposiciones sociales presentes en el Otro social. Se podría aceptar, en consecuencia, que el momento adolescente puede ser concebido como un espacio de traducción de discursos, y por ello, como un momento de interrogación respecto de la legitimidad de las referencias parentales, familiares y sociales. Así comprendido, el trabajo de la singularidad de la adolescencia, vendría a conformar un espacio de encuentro con lo real que permitirá, a partir de su carácter intraducible, sobrepasar el impase entre el mundo familiar, los grupos de referencia y el Otro social. De hecho, es posible inferir que es a partir de la imposibilidad de traducción presente en la experiencia adolescente, en donde se radica la posibilidad de donar nuevas formas para transmitir la inestabilidad del deseo y el carácter siempre inacabado de los esfuerzos de historización.

\section{A modo de conclusión}

De acuerdo a la hipótesis que se ha intentado examinar, los mecanismos de deconstrucción del orden social instituyente durante el momento adolescente, permiten el reposicionamiento del sujeto frente al Otro social. Siendo este despliegue de la subjetividad caracterizado por el carácter inestable y la impropiedad de los esfuerzos de simbolización. A la luz de lo anterior es factible observar un cierto número de fenómenos que explicitan al menos, los siguientes criterios de análisis, a) la Ley: el pasaje del Otro parental al Otro social, otorga la posibilidad de diferenciar la ley social de la Ley del lenguaje, es decir, permite comprender la historicidad y el carácter contingente de la ley social y admitir la instancia del lenguaje como constituyente de la condición humana, b) el Súper yo: el Otro social, recubierto de las potestades del Otro paterno, vendrá a conformar una discursividad, que el adolescente experimenta como vivencia psíquica tanto a nivel consciente como inconsciente, y respecto de la cual, el sujeto adolescente conformará diferentes estrategias de subjetivación, tanto a nivel de lo sexual como de los ideales sociales, c) lo Real: el encuentro con lo real en el plano de la sexuación, conforma una apertura a la falta que orienta el trabajo de la sublimación y la ética del sujeto-adolescente, el cual se abre a nuevas formas de posicionamiento respecto de 
la Ley, dando lugar a procesos de narrativización y transmisión psíquica, d) el Lazo social: la conformación de vínculos sociales, vale decir, la apertura a la alteridad y a la responsabilidad respecto de otros, instituye y da legitimidad a nuevas formas de analizar el deseo y las actuaciones del sujeto-adolescente, e) el Discurso: la validación del Nombre-del-padre, en tanto que nombres-delpadre durante la adolescencia, remarca la necesidad de inscribir la aceptación de la falta a nivel de los vínculos y de las prácticas sociales, f) lo Imposible: el reconocimiento de la falta como constituyente de la experiencia ética del sujeto, pero también de su experiencia social a nivel del discurso, demanda el reconocimiento del carácter provisorio de las construcciones humanas, reafirmando la historicidad del lazo social desde los procesos de historización que emergen en el momento adolescente de subjetivación.

Dicho de otra forma, dentro del tiempo y el espacio de las simbolizaciones de la adolescencia, el sujeto se abre a la posibilidad de comprender que dentro de la inestabilidad y la contingencia de toda ley, él mismo esta llamado a reencontrar el fundamento de la Ley en tanto que representante de excepción, y por excepción, del propio deseo. Se trata pues, de visualizar el momento adolescente como un proceso de reconocimiento del vacío inherente

446 a la cadena de los significantes, de suerte que a este nivel, se prueba el valor solamente simbólico del significante de la Ley y la inevitable singularidad del deseo frente a lo real. Asimismo, se trata de pensar las posibilidades abiertas por la dialéctica adolescente, desde una perspectiva capaz de subrayar la importancia del reencuentro con el otro dentro del proceso de construcción de narraciones capaces de ocupar el espacio de transición entre los diferentes miembros de un grupo social, el conjunto de la comunidad y de transmisión entre las generaciones.

Se estima en consecuencia posible sostener, que el estudio de los mecanismos de deconstrucción del orden social instituyente, viene a ocupar la plaza de una interrogante fundamental dentro de la clínica contemporánea de la adolescencia. De suerte que el estudio de la posibilidad de deconstruir la Ley durante el momento adolescente, se configura como un elemento teóricoclínico, que permite diferenciar la sumisión a los mandatos súperyoicos del Otro social o del Otro grupal, de la posibilidad de singularizar la posición del joven y el adolescente frente a estas exigencias. De esta forma, esta visualización del momento adolescente permite también generar una herramienta de interpretación frente al incremento de los procesos de radicalización y de sobre identificación imaginaria, presentes, de modo cada vez más evidente, entre jóvenes y adolescentes en las sociedades contemporáneas. 


\section{ARTIGOS}

\section{Referências}

Brackelaire, J. L. (1995). La personne et la société. Bruxelles, Belgique: De Boeck Université.

Douville, O. (2014). Les figures de l'Autre. Paris, France: Dunod.

Dufour, D. R. (2003). L'art de réduire les têtes. Paris, France: Denoël.

Forget, J. M. (2005). L'adolescent face à ses actes... et aux autres. Une clinique de l'acte. Ramoville Saint-Agne, France: Éditions Érès.

Freud, S. (1978). Proyecto de psicología. In J. Strachey (Ed.) y J. L. Etcheverry (Trad.), Obras completas: Sigmund Freud (Vol. I). Buenos Aires, Argentina: Amorrortu. (Trabajo original publicado en 1950 [1895]).

Freud, S. (1978a). El yo y el ello. In J. Strachey (Ed.) y J. L. Etcheverry (Trad.), Obras completas: Sigmund Freud (Vol. XIX). Buenos Aires, Argentina: Amorrortu. (Trabajo original publicado en 1923-25).

Freud, S. (1978b). Psicología de las masas y análisis del yo. In J. Strachey (Ed.) y J. L. Etcheverry (Trad.), Obras completas: Sigmund Freud (Vol. XVIII). Buenos Aires, Argentina: Amorrortu. (Trabajo original publicado en 1921).

Freud, S. (1978c). El malestar en la cultura. In J. Strachey (Ed.) y J. L. Etcheverry (Trad.), Obras completas: Sigmund Freud (Vol. XXI.) Buenos Aires, Argentina Amorrortu. (Trabajo original publicado en 1920).

Gagnepain, J. (1991). Du vouloir dire II. De la personne. De la norme. Paris, France: Livre et communication.

Gagnepain, J. (2010). Huit Leçons d'Introduction à la Théorie de la Médiation. Recuperado de: $<$ http://www.institut-jean-gagnepain.fr/huit-leçons-d-introductionà-la-théorie-de-la-médiation/Institut Jean Gagnepain>.

Gauchet, M. (2009). Personne, individu, sujet, personnalité. En Marcel Gauchet \& Jean Claude Quentel (Comp.), Histoire du sujet et théorie de la personne (pp.11-41). Rennes, France: Presses Universitaires de Rennes.

Gutton, Ph. (2002). Violence et adolescence. Paris, France: In Press Éditions.

Haie B. y Douville, O. (2007). Le mathème du fantasme à l'adolescence. Psychologie française, 52, 123-136.

Haie B. y Rassial, J. J. (2008). L'adolescence : moment de construction du sinthome ou de refonte du fantasme. Adolescence, 22, 237- 247

Lacan, J. (1966). Subversion du sujet et dialectique du désir dans l'inconscient freudien. En Écrits (Vol. I, pp. 793-828). Paris, France: Seuil.

Lacan, J. (1973). Le séminaire. Livre XI. Les quatre concepts fondamentaux de la psychanalyse. Paris, France: Seuil. (Trabajo original publicado en 1964). 


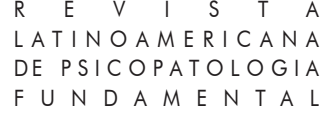

Lacan, J. (1986). Le séminaire. Livre VII, L'éthique. Paris, France: Seuil. (Trabajo original publicado en 1959-1960).

Lacan, J. (2010). Le séminaire. Livre IX, L'Identification. Paris, France: Éditions de l'association lacanienne internationale. (Trabajo original publicado en 1961-1962)

Lesourd, S. (2002). Adolescence... Rencontre avec le féminin. Ramonville SaintAnge, France: Érès.

Lesourd, S. (2004). La déconstruction-reconstruction des systèmes référentiels. En Ph. Gutton et Stéphane Bourcet (Comp.), Naissance pubertaire (pp. 99-126). Paris, France: Denoël.

Lesourd, S. (2006). Comment taire le sujet? Des discours aux parlottes libérales. Ramonville Saint-Agne, France: Érès.

Mijolla-Mellor, S. (2009). Le choix de la sublimation. Paris, France: Presses Universitaires de France.

Mijolla-Mellor, S. (2012). Traité de la sublimation. Paris, France: Presses Universitaires de France.

Pirard, R. (2013). L'époque et le sujet. Psychanalyse, (26), 101-121.

Quentel, J. C. (2011). L'adolescence aux marges du social. Bruxelles, Belgique: Yapaka.

Rassial, J. J. (1996) Le passage adolescent. Toulouse, France: Érès.

Rassial, J. J. (1999). Le sujet en état limite. Paris, France: Denoël.

Saint-Cyr, V. (2012). Créer un vide ou de la sublimation chez Lacan. Recherches en Psychanalyse, 13,14-2.

Sauret, M. J. (2008). L'effet révolutionnaire du symptôme. Ramonville Saint-Agne, France: Érès.

Sauret, M. J. (2009). Adolescence et lien social : le moment adolescent. Adolescence, 68, 313-327.

\section{Resúmenes}

(Sobre os mecanismos de desconstrução da ordem social instituída durante a adolescência)

Os estudos desenvolvidos sobre a questão do supereu na adolescência reconhecem a possibilidade e a necessidade de distanciamento do individuo frente a esta aparência, deixando, ainda assim, abertas as perguntas sobre os mecanismos desta operação. Neste artigo, é proposta uma leitura dessa problemática, mediante a análise 


\section{ARTIGOS}

do vínculo entre o trabalho do posicionamento adolescente em relação à ordem social instituida e o trabalho da sublimação no indivíduo. Com esse propósito, a proposta será compreender as possibilidades de a pessoa posicionar-se eticamente frente à realidade, a partir da construção de um espaço subjetivo de transição, interpretação e narração.

Palavras-chave: Ética, sublimação, ordem social instituída, singularidade

(Regarding the mechanisms that deconstruct established social order during adolescence)

Studies about the superego in adolescence acknowledge the possibility and need to distance individuals from this appearance; nevertheless, they leave certain questions unanswered regarding the mechanisms inherent to this behavior. This paper proposes a review of this issue through analysis of the bond between the process of teenage positioning regarding established social order and the process of sublimation in individuals. To this end, we suggest understanding individuals' ability to position themselves ethically facing reality, based on the establishment of a subjective place of transition, interpretation and narration.

Key words: Ethics, sublimation, established social order, singularity

(À propos des mécanismes de déconstruction de l'ordre social institué au moment de l'adolescence)

Les études menées autour de la question du surmoi lors de l'adolescence reconnaissent la possibilité et le besoin d'établir une distance entre le sujet et ce concept, laissant néanmoins ouvertes les questions relatives aux mécanismes régissant cette manoeuvre. Cet article propose une lecture de cette problématique par l'analyse du lien entre le travail de positionnement de l'adolescent face à l'ordre social instituant et le travail de la sublimation chez le sujet. C'est dans ce but que notre propos vise à comprendre les possibilités du sujet de se positionner de manière éthique face au réel à partir de la construction d'un espace subjectif de transition, de traduction et de narration.

Mots clés: Éthique, sublimation, ordre social instituant, singularité

(Mechanismen der Deskonstruktion der bestehenden gesellschaftlichen Ordnung in der Adoleszenz)

Die Studien, die sich mit dem Über-Ich in der Adoleszenz befassen, bestätigen die Möglichkeit und Notwendigkeit der Distanzierung des Individuums vom Über-Ich, lassen jedoch Fragen zu den Mechanismen dieses Vorgangs offen. Dieser Artikel beschreibt einen Ansatz zu diesem Problem, wobei der Zusammenhang zwischen der Positionierung des Jugendlichen innerhalb der bestehenden gesellschaftlichen Ordnung und der Sublimierung des Subjekts analysiert wird. Dieser Ansatz ermöglicht es uns, die allfälligen ethischen Stellungnahmen des Individuums zur Realität 
zu verstehen, wobei zu diesem Zweck ein subjektiver Raum für den Übergang, die Auslegung und die Narration geschaffen wird.

Schlüsselwörter: Ethik, Sublimation, bestehende gesellschaftliche Ordnung, Singularität

Citação/Citation: Bilbao, A., \& Jofré, D. (2017, setembro). Acerca de los mecanismos de deconstrucción del orden social instituyente durante el momento adolescente. Revista Latinoamericana de Psicopatologia Fundamental, 20(3), 436-450. http://dx.doi.org/10.1590/ $1415-4714.2017 \mathrm{v} 20 \mathrm{n} 3 \mathrm{p} 436.2$

Editores do artigo/Editors: Profa. Dra. Ana Maria Rudge e Profa. Dra. Sonia Leite.

Recebido/Received: 20.5.2016/ 5.20.2016 Aceito/Accepted: 9.9.2016/9.9.2016

Copyright: (C) 2009 Associação Universitária de Pesquisa em Psicopatologia Fundamental/ University Association for Research in Fundamental Psychopathology. Este é um artigo de livre acesso, que permite uso irrestrito, distribuição e reprodução em qualquer meio, desde que $\mathrm{o}$ autor e a fonte sejam citados / This is an open-access article, which permits unrestricted use, distribution, and reproduction in any medium, provided the original authors and sources are credited.

450 Financiamento/Funding: Os autores declaram não ter sido financiados ou apoiados. The authors have no support or funding funded to report.

Conflito de interesses/Conflict of interest: Os autores declaram que não há conflito de interesses / The authors have no conflict of interest to declare.

\section{Alejandro Bilbao}

Director programa de Doctorado en Psicoanálisis.

Facultad de humanidades y Ciencias Sociales Andrés Bello

Fernandez Concha 700, Las Condes

Santiago de Chile, Chile (Cód. Postal 7591538)

bilbao1231@hotmail.com

\section{Daniel Alejandro Jofré Astudillo}

Doctor en Recherches en Psychanalyse et Psychopathologie, Université Paris 7 - Diderot (Paris, France); Profesor de Psicopatología y de Psicología clínica de la infancia y la adolescencia, Universidad Austral de Chile (Puerto Montt, Chile).

Universidad Austral de Chile

Casilla N 1327

Puerto Montt, Chile.

jofre.daniel@gmail.com / daniel.jofre@uach.cl

This is an open-access article, which permits unrestricted use, distribution, and reproduction in any medium for non-commercial purposes provided the original authors and sources are credited. 